\title{
Turbulent characteristics of flow in rotating cylinders
}

\author{
Zhongfan $\mathrm{ZHU}^{1,}$, and Ying WANG ${ }^{2, b}$ \\ ${ }^{1}$ College of Water Sciences, Beijing Normal University, Xinjiekouwai Street 19, Beijing 100875, \\ China \\ ${ }^{2}$ Fengtai No.2 Middle School, Fengtai District, 100071, Beijing, China \\ azhuzhongfan1985@bnu.edu.cn, blinda_wang0205@126.com
}

Keywords: Cylinders; Turbulent characteristics; ADV; Flow shear rate

Abstract. Based on measured data of velocities of several points along the vertical direction in the center of rotating cylinders using Acoustic Doppler Velocimeter (ADV), we analyzed some turbulent characteristics of flow, such as time-averaged velocity, turbulent intensity, and Reynolds stress. In addition, we also estimated the flow shear rate, a parameter used to characterize the shear flow.

\section{Introduction}

Experimental investigation on the particle movement in the estuarine and coastal water has a great engineering value. Three kinds of devices were commonly used to model an actual flow shear environment in some studies [1-3]: an impeller mixer, an oscillating grid and the rotating cylinders. Among them, the rotating cylinder apparatus (where the flow pattern has been termed to be the Couette-flow system) shows its unique characteristics since it can generate a more isotropic shear flow when compared with other devices [2]. However, insufficient information about the flow filed in the rotating cylinders have been found, and the aim of this study is to analyze the turbulent characteristics of flow filed, such as time-averaged velocity, turbulent intensity and Reynolds stress, based on measured data of velocities of several points along the vertical direction in the center of rotating cylinders using ADV.

\section{Experimental device}

Fig. 1 shows a schematic of the experimental device. The device consists of an inner rotating cylinder with a radius, $R_{1}$, of $150 \mathrm{~mm}$, and an outer stationary cylinder with a radius, $R_{2}$, of $236 \mathrm{~mm}$. The height of both cylinders is $682 \mathrm{~mm}$, and the water depth, $h$, kept at approximately $400 \mathrm{~mm}$ during the experiments (in view of the super-elevation of the water surface that resulted from an eccentric motion). The cylindrical coordinate system is also shown in Fig.1, where the tangential direction is demoted by $\theta$, the radial one by $r$, and the vertical by $z$ calculated from the bottom of the cylinders. A speed-adjusting motor is responsible to control the angular velocities of the inner cylinder.

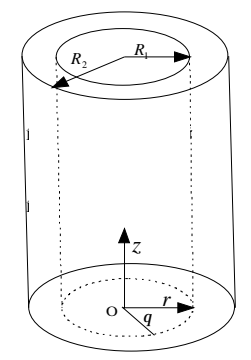

Fig.1. Schematic of the rotating cylinders.

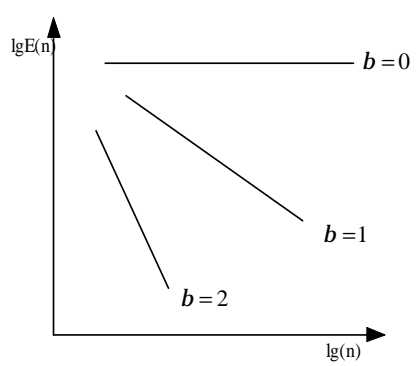

Fig. 2. Some frequency spectrum patterns.

As the rotating velocity of the inner cylinder increased, an ADV (with the velocity range from $1 \mathrm{~mm} / \mathrm{s}$ to $2.5 \mathrm{~m} / \mathrm{s}$, and accuracy $0.1 \mathrm{~mm} / \mathrm{s}$ with a standard deviation of $1 \%$; produced by SonTek Corporation, USA) was used to measure the flow filed in rotating cylinders. Three ADV probes, each $38.5 \mathrm{~mm}$ in 
length, can measure the tangential, radial and vertical components of the velocity respectively. Because of the limitations of the ADV dimension and the cylinders, we only measured the velocities of some points along the vertical direction in the center of the apparatus. The number of measured points is 8 or 9. Temperature during the experiments kept at about $20^{\circ} \mathrm{C}$.

\section{Turbulent characteristics of flow field}

Determination of modes of flow. Since the aim of this study is to investigate the turbulent characteristics of flow field, it is necessary to determine the modes of flow in the rotating cylinders as the rotating velocity of the inner cylinder, $\omega$, increases. The flow filed in the rotating cylinders has been termed as the Taylor-Couette flow[4]. During the experiments, we used the method of the power spectrum analysis to determine the modes of flow. The principle of the power spectrum analysis is briefly introduced as follows. The time series of instantaneous velocity, $U$, can be transformed to be frequency series using Fourier transformation as expressed as follows:

$$
G(n)=\sum_{k=0}^{N-1} U(k) e^{-j \frac{2 \pi n}{N} k}, n=0,1,2, \cdots N-1 .
$$

where $U(k)$ is time series of the fluctuating velocity ( $k$ is the number index, and it increases from 0 to $N-1$, where $N$ is the number of sampling), $G(n)$ is Fourier transformation ( $n$ is the number index of the frequency spectrum, and it ranges from 0 to $N-1$ ), the frequencies are $n / N T_{s}, T_{s}$ is time interval of sampling (here it equals to $25 \mathrm{~Hz}$ ), and $j$ is the imaginary unit in the complex. The power density spectrum $E(n)$ is:

$$
E(n)=\frac{2 T_{s}}{N} G(n) * G^{*}(n), n=0,1,2, \cdots N-1 .
$$

where $G^{*}(n)$ is the conjugate complex of Fourier transformation $G(n)$. When the $E(n)$ and $n$ data are plotted in the double-log coordinate system, the slope of the curve, $\beta$, can be used to study the frequency spectrum properties of flow filed. As shown in Fig.2, the line of $\beta=0$ represents the frequency spectrum of white noise, the line of $\beta=1$ the frequency spectrum of $1 / n$ noise, and the line of $\beta=2$ denotes the frequency spectrum of Brownian noise[5]. Whereas for the isotropic turbulent flow, $\beta$ should be 5/3[6]. We analyzed the the frequency spectrum properties as the rotating velocity of the inner cylinder $\omega$ increases from 0.03 to 3 revolutions per second (rps). Table 1 presents these results, and it can be found that the flow field becomes the turbulent flow as long as $\omega$ is larger than the critical one, $\omega_{c}(=0.45 \mathrm{rps})$. As a result, the range of $\omega$ from 0.5 to $3 \mathrm{rps}$ was chosen in this study.

Table 1. Results of the frequency spectrum analysis.

\begin{tabular}{cccc}
\hline$\omega(r p s)$ & Results & $\omega(r p s)$ & Results \\
\hline 0.03 & White noise & 0.6 & Turbulence \\
0.06 & White noise & 0.7 & Turbulence \\
0.08 & White noise & 1 & Turbulence \\
0.1 & White noise & 1.5 & Turbulence \\
0.3 & White noise & 2 & Turbulence \\
0.4 & White noise & 2.5 & Turbulence \\
0.45 & Turbulence & & Turbulence \\
0.5 & Turbulence & \\
\hline
\end{tabular}

Time-averaged velocity. Fig.3 (a)-(c) shows the tangential, radial and vertical time-averaged velocity components, $\overline{u_{\theta}}, \overline{u_{r}}, \overline{u_{z}}$, respectively, as the rotating velocity of the inner cylinder, $\omega$, increases from 
0.5 to 3rps. A uniform distribution of $\overline{u_{\theta}}$ along the vertical direction except at the bottom of cylinders can be seen in Fig. 3(a). It can be found from Fig. 3(b) that a clockwise circulation takes place in the flow field when $\omega$ is between 1 and $3 \operatorname{rps}$ in terms of $\overline{u_{r}}$, that is, fluid particles in the region of $z / h>0.1$ flow toward the outer cylinder, whereas rest fluid particles flow toward the inner cylinder. However, this circulation does not happen when $\omega$ is between 0.5 and $0.7 \mathrm{rps}$, for both cases, the velocity changes greatly along the vertical direction. From Fig. 3(c), it can be observed that $\overline{u_{z}}$ is small at the water surface and the bottom of the cylinders, and it reaches the peak value at the specific point of the middle region. For the cases of $\omega=0.5,0.6$ and $0.7 \mathrm{rps}$, the fluid particles in the region of $z / h>0.2$ flow downwards, whereas rest fluid particles moves upwards. However, for the cases of $\omega=1,1.5,2,2.5$ and 3rps, fluid particles in the region of $z / h>0.7$ move upwards, while rest ones flow downwards. These movement characteristics may improve the formation of an isotropic turbulence flow, and should play an important role in the convection and dispersion processes of particles in the cylinders.

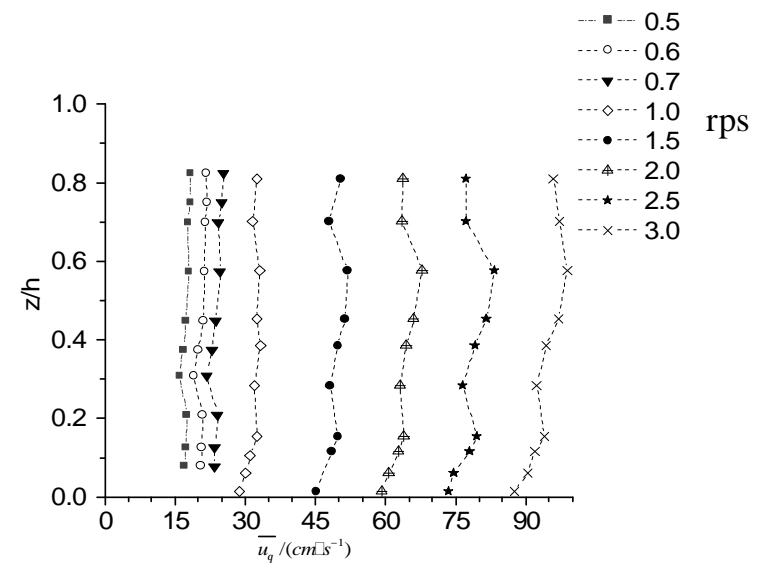

(a)

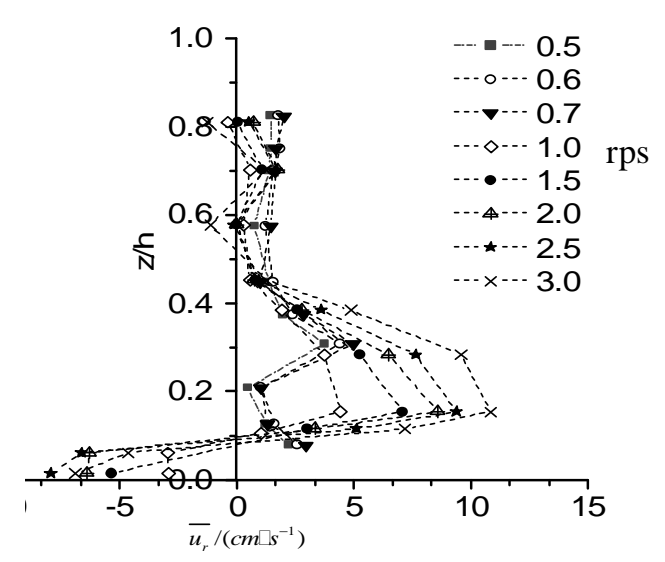

(b)

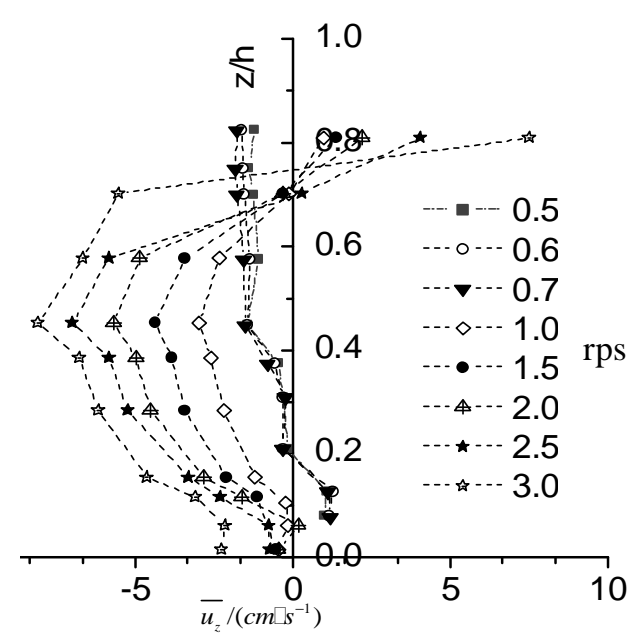

(c)

Fig.3. Tangential (a), radial (b) and vertical (c) time-averaged velocity components.

Turbulent intensity. Tangential, radial and vertical turbulent intensity components, $u_{\theta}^{\prime}, u_{r}^{\prime}, u_{z}^{\prime}$, are calculated as: $u_{\theta}^{\prime}=\sqrt{\overline{u_{\theta}^{2}}}, u_{r}^{\prime}=\sqrt{\overline{u_{r}^{2}}}, u_{z}^{\prime}=\sqrt{\overline{u_{z}^{2}}}$, where $u_{\theta}, u_{r}, u_{z}$ are tangential, radial and vertical fluctuating velocity components. Fig. 4(a)-(c) shows the distributions of $u_{\theta}^{\prime}, u_{r}^{\prime}, u_{z}^{\prime}$ (divided by the velocity of inner cylinder, $\omega R_{1}$ ) along the vertical direction as $\omega$ increases from 1 to 3rps. It can be seen that $u_{\theta}^{\prime}, u_{r}^{\prime}$ and $u_{z}^{\prime}$ keep approximately uniform along the vertical direction in the region of 
$0.17<z / h<0.7$, despite of some small variations along the vertical direction. Whereas $u_{\theta}^{\prime}, u_{r}^{\prime}$ and $u_{z}^{\prime}$ change significantly in the region near the bottom of cylinders $(z / h<0.17)$, and they also vary greatly in the region near the water surface $(z / h>0.7)$. The peak of three turbulent intensity components appears at the position of $z / h \approx 0.17$. Additionally, $u_{\theta}^{\prime}, u_{r}^{\prime}$ and $u_{z}^{\prime}$ increase as the angular velocity of the inner cylinder, $\omega$, improves. The reason why there is an approximately uniform distribution of the turbulent intensity in the middle region of flow filed $(0.17<z / h<0.7)$ is that the clockwise circulation as presented in section 3.2 promotes the mixing among fluid particles, consequently the development of turbulence toward a uniform one. However, this mixing may not play a significant role in the region near the bottom $(z / h<0.17)$ because of non-slip condition and another region near the water surface $(z / h>0.7)$ due to free fluctuating influence, as a result the turbulent intensity changes greatly in these regions.

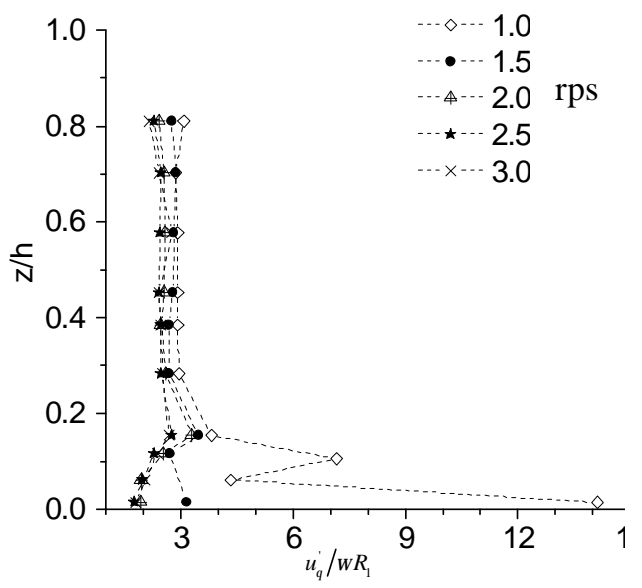

(a)

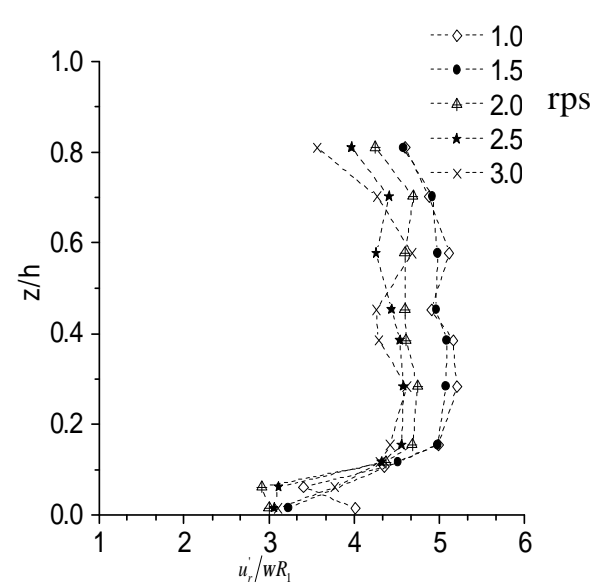

(b)

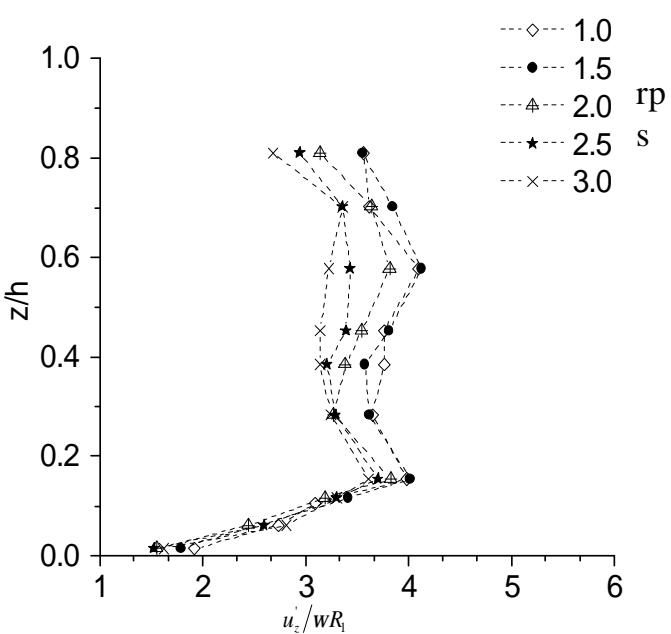

(c)

Fig. 4. Tangential (a), radial (b) and vertical (c) turbulent intensity components.

Probability distribution of the fluctuating velocity. Kurtosis coefficients, $\gamma_{\theta}, \gamma_{r}, \gamma_{z}$, are three parameters that could be used to characterize the peak degree of vertical distributions of tangential, radial and vertical fluctuating velocity components respectively. They are defined as: $\gamma_{\theta}=E\left(u_{\theta}{ }^{4}\right) / \sigma_{\theta}{ }^{4}, \gamma_{r}=E\left(u_{r}{ }^{4}\right) / \sigma_{r}{ }^{4}, \gamma_{z}=E\left(u_{z}{ }^{4}\right) / \sigma_{z}{ }^{4}$, where $E\left(u_{\theta}{ }^{4}\right), E\left(u_{r}{ }^{4}\right)$ and $E\left(u_{z}{ }^{4}\right)$ are fourth-order moments of three fluctuating velocity components, and $\sigma_{\theta}, \sigma_{r}$ and $\sigma_{z}$ are standard deviations of their distribution curves, respectively. The value $\gamma=3$ represents a normal distribution, $\gamma>3$ being a positive distribution, whereas the value $\gamma<3$ shows a negative one. Fig. 5 (a)-(c) shows 
vertical distributions of $\gamma_{\theta}, \gamma_{r}$ and $\gamma_{z}$ when $\omega$ increases from 1 to 3 rps. Most of the $\gamma_{\theta}$ values are almost near to 3 except for those at some specific positions. In the region $(z / h>0.15)$, the $\gamma_{r}$ values are almost near to 3 , showing that the $u_{r}$ has a normal distribution; whereas in the region near the bottom $(z / h<0.15)$, the $u_{r}$ experiences a gradual increase to a peak value before finally decreasing to 3 with the increasing distance approaching the bottom of cylinders, representing a positive distribution of the $u_{r}$. In the region $(z / h>0.25)$, the vertical distribution of the $\gamma_{z}$ is approximately uniform, and their values are near to 3 . However, in the region near the bottom $(z / h<0.15)$, the $\gamma_{z}$ value increases rapidly with the increasing distance approaching the bottom, showing that $u_{z}$ has a positive distribution.

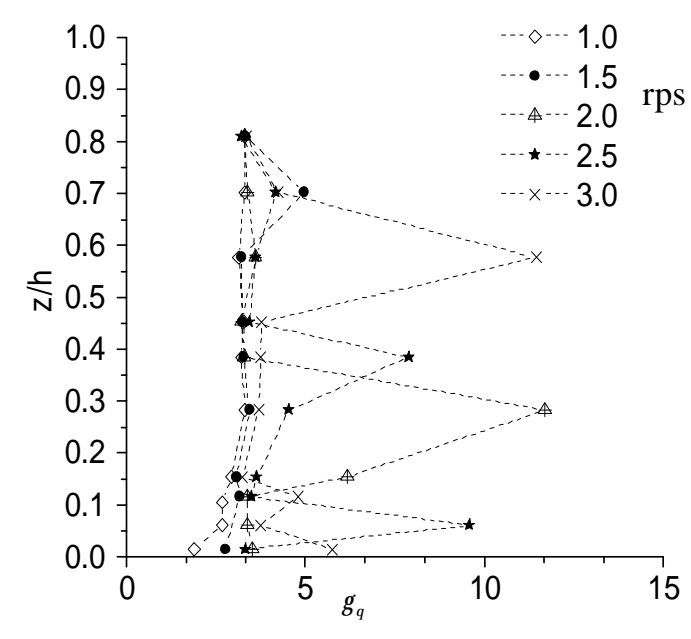

(a)

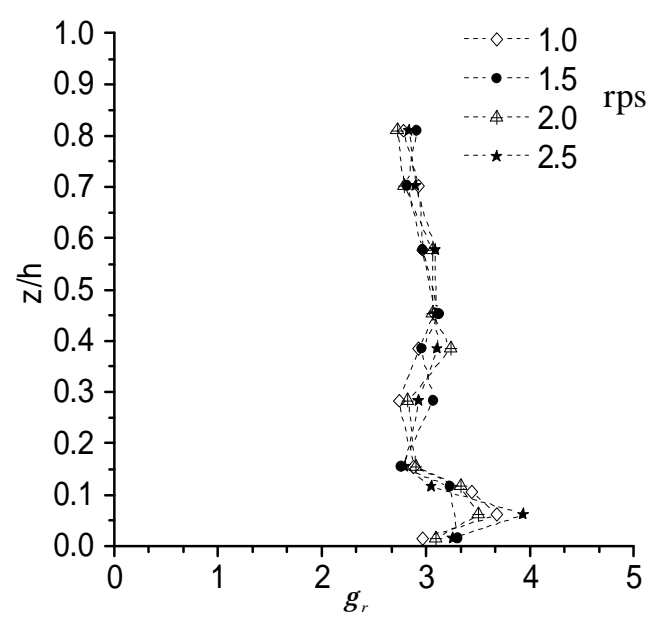

(b)

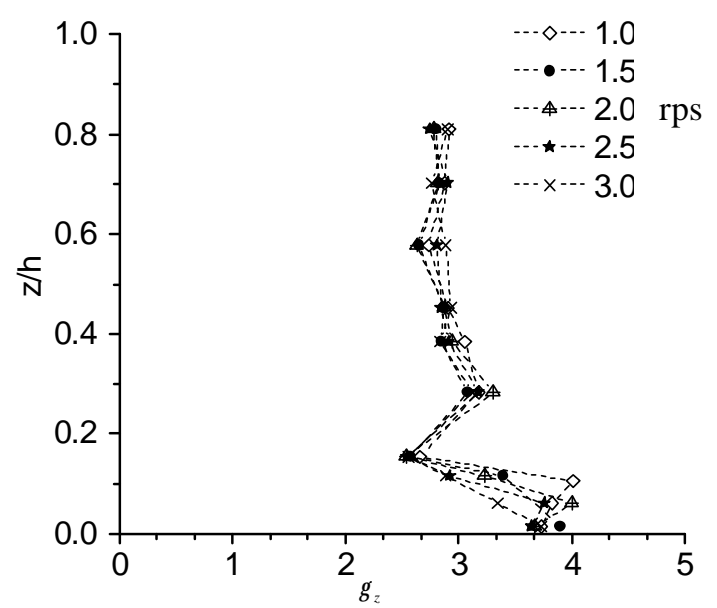

(c)

Fig. 5. Kurtosis values of the distributions of tangential (a), radial (b) and vertical (c) fluctuating velocity components.

Reynolds stress. The radial-tangential, vertical-tangential and vertical-radial Reynolds stress components, denoted by $\tau_{r \theta}, \tau_{z \theta}$ and $\tau_{z r}$, are defined as: $\tau_{r \theta}=-\rho \overline{u_{r} u_{\theta}}, \tau_{z \theta}=-\rho \overline{u_{z} u_{\theta}}, \tau_{z r}=-\rho \overline{u_{z} u_{r}}$, where $\rho$ is the density of water, and the bar is taken as a time-averaged quantity, respectively. Fig. 6 (a)-(c) shows vertical distributions of $\tau_{r \theta}, \tau_{z \theta}$ and $\tau_{z r}$ when $\omega$ improves from 1 to 3rps. The vertical distribution of $\tau_{r \theta}$ is uniform in the region $(z / h>0.2)$ except for the case of $\omega=3$ rps. However, the $\tau_{r \theta}$ experiences an abrupt variation in the region near the bottom of cylinders $(z / h<0.15)$ regardless 
of $\omega$. The $\tau_{z \theta}$ and $\tau_{z r}$ vary significantly across the height of cylinders, as shown in Fig. 6 (b)-(c) respectively.

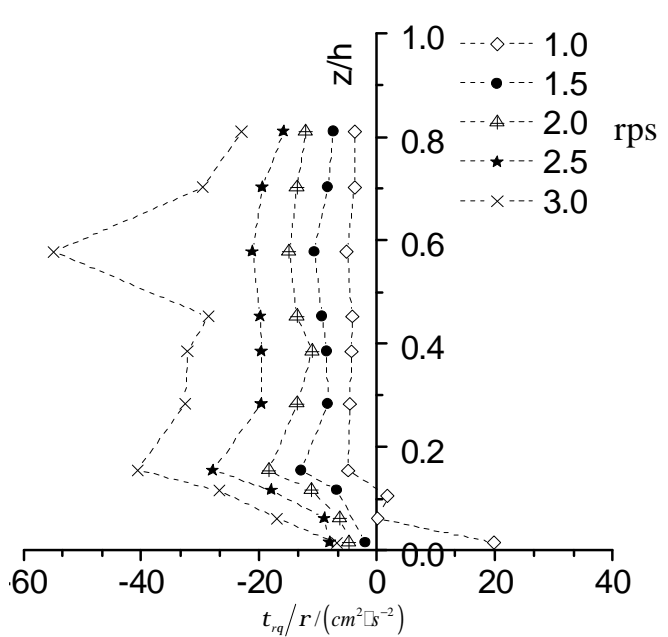

(a)

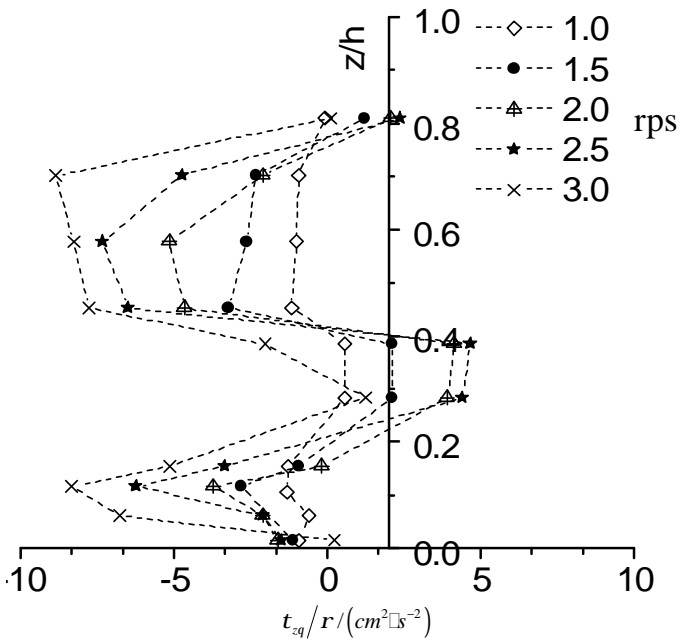

(b)

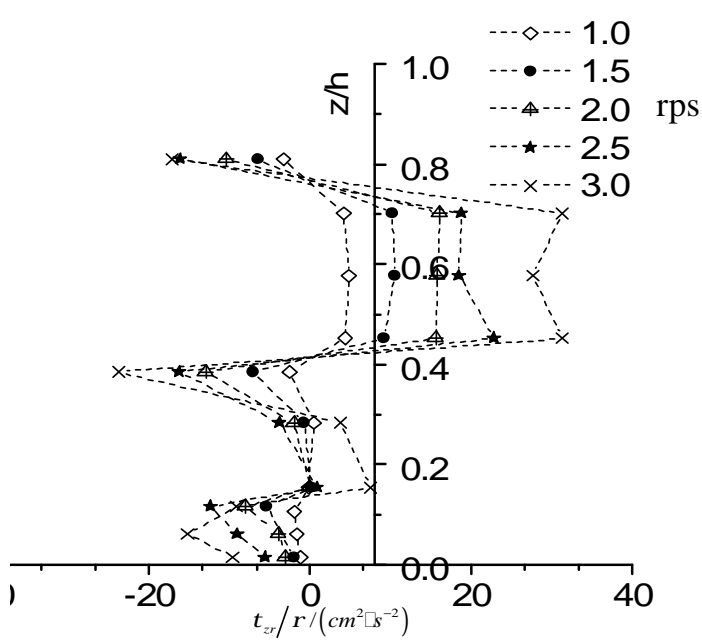

(c)

Fig. 6. Radial-tangential (a), vertical-tangential (b) and vertical-radial (c) Reynolds stress components.

\section{Estimation of the shear rate}

In most of rotating cylinder experiments, the shear rate (or the shear velocity gradient), $G$, has been used to describe the shear flow [2]. This parameter has been estimated using two different methods, depending on whether the flow is laminar or turbulent. For laminar flow, the shear rate, $G$, the average value of the gradient of the tangential instantaneous velocity component along the axial direction across the gap of cylinders in the cylindrical coordinate system, was calculated by using the following expression [2]

$$
G=\frac{2 \omega R_{1} R_{2}}{R_{2}^{2}-R_{1}^{2}}
$$

Whereas for turbulent flow, the $G$ can be estimated as follows [7]

$$
G=\sqrt{\frac{\varepsilon}{v}}
$$

where $\varepsilon$ is the energy dissipation rate per unit mass $\left(\mathrm{m}^{2} / \mathrm{s}^{3}\right)$ and $v$ is the kinematic viscosity of water $\left(\mathrm{m}^{2} / \mathrm{s}\right)$. It seems difficult to estimate the exact value for the rate of the energy dissipated into the system, $\varepsilon$, and in this study, we used the method presented by Serra et al. (1997)[3]. In their method, $\varepsilon$ is 
assumed to scale as $\varepsilon \propto u^{\prime 3} / I$ when using a characteristic velocity fluctuation, $u^{\prime}$, and a characteristic length, $I$. Furthermore, it was assumed that the value of $I$ is scaled to the width of the gap, $\mathrm{I} \propto R_{2}-R_{1}$. Here it seems more reasonable to use the averaged turbulent intensity measured by ADV, $\sqrt{\left(u_{\theta}^{\prime 2}+u_{r}^{\prime 2}+u_{z}^{\prime 2}\right) / 3}$, to replace the value of $u^{\prime}$. This method was used instead of the method of Serra et al. where the $u$ ' value was simply assumed to scale to the mean velocity, which is a specific function of the angular velocity of the inner cylinder. However, if continuity between the laminar shear rate (calculated based on Eq. (3)) and the turbulent shear rate (calculated based on Eq. (4) and some scaling relations) at the critical angular velocity, $\omega_{c}$ (presented in section 3.1), for every measured point is assumed, a proportionality ratio can be obtained. Once this ratio was determined, the turbulent shear rate values corresponding to different angular velocities of the inner cylinder which exceed the critical value could be finally estimated. Fig. 7 shows vertical distributions of $G$ values as $\omega$ improves from 0.5 to 3rps. The larger the $\omega$ value is, the higher the $G$ value is. In the region near the bottom of cylinders $(z / h<0.15)$, the $G$ value changes significantly and reaches the peak value at the position of $z / h=0.15$. The vertical distribution of the $G$ value is approximately uniform for the cases of $\omega=0.5,0.6,0.7,1.0$ and 1.5rps in the rest region $(z / h>0.15)$, whereas for the cases of $\omega=2.0,2.5$ and 3.0rps, a noticeable vertical variation of the $G$ value can be observed in this region.

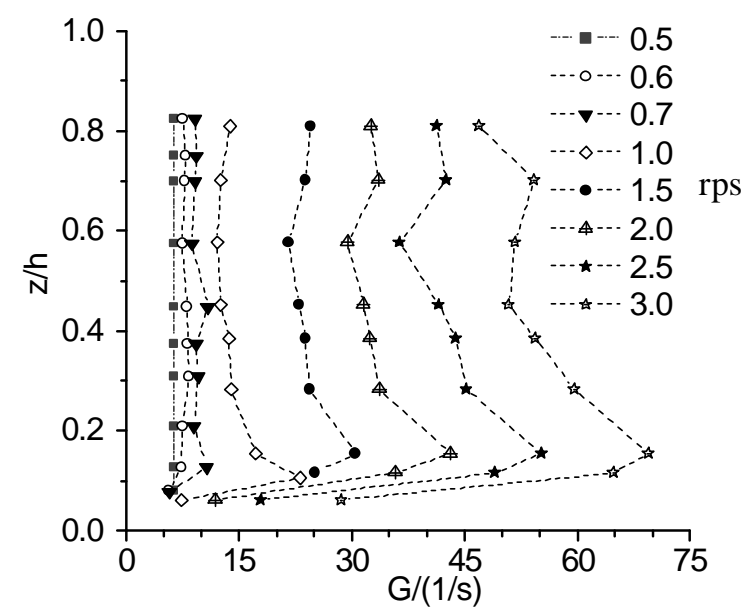

Fig. 7. Vertical distributions of shear rate values with the increasing velocity of the inner cylinder (the unit is rps).

\section{Concluding remarks}

In this study, we measured the velocities of some points along the vertical direction in the center of the gap of rotating cylinders (it consists of an inner cylinder rotating with different angular velocities and a fixed outer cylinder) using ADV.

Based on the measured data, we analyzed the vertical distributions of some parameters in rotating cylinders, and these parameters include the time-averaged velocity, the turbulent intensity, the probability distribution of the fluctuating velocity and Reynolds stress. The results indicated that the flow field showed different turbulent characteristics in different regions: near-bottom region $(z / h<0.15)$, middle region $(0.15<z / h<0.7)$ and near-free surface region $(z / h>0.7)$.

Finally, we estimated the shear rate values corresponding to different angular velocity of the inner cylinder. These results provide some information about flow field in the Taylor-Couette flow.

\section{Acknowledgements}

This work was financially supported by the Fundamental Research Funds for the Central Universities in China (No:2013NT50, 2015KJJCB16) and the project Sponsored by the Scientific Research Foundation for the Returned Overseas Chinese Scholars, State Education Ministry. 


\section{References}

[1] C. Biggs and P. Lant: Water Res. Vol.34 (2000), pp.2542.

[2] T. Serra, J. Colomer and B.E. Logan: Water Res. Vol.42 (2008), pp.1113.

[3] T. Serra, J. Colomer and X. Casamitjana: J. Colloid Interface Sci. vol.187 (1997), p.466

[4] R.C.Di Prima and H.L. Swinney, in: Hydrodynamic Instabilities and the Transition to Turbulence, volume 45 of the series Topics in Applied Physics, chapter, 6, Springer-Verlag Publishers (1985).

[5] X. Sun and Z, Wu: The principles of fractal theory and its application (University of Science and Technology of China Press, China 2003) (in Chinese)

[6] J.O.Hinze: Turbulence (McGraw Hill, USA 1975).

[7] T.R. Camp and P.C. Stein: J. Boston Soc. Civil Eng. Vol.85 (1943), p. 219 